\title{
Effect of Normal Saline Bicarbonate on Reversing Epidural Anesthesia Either with Bupivacaine Or Bupivacaine and Nalbuphine in Patients Undergoing Orthopedic Surgery
}

\author{
Josef Attia, Ahamed Mohamed, and Yaser Bader \\ Departments of Anesthesiology and ICU, Faculty of Medicine, Al-Minia University, Minia 61111, Egypt \\ Correspondence should be addressed to Josef Attia; josefzekry2@yahoo.com
}

Received 27 September 2014; Revised 7 February 2015; Accepted 16 February 2015

Academic Editor: Young-Chang P. Arai

Copyright ( 2015 Josef Attia et al. This is an open access article distributed under the Creative Commons Attribution License, which permits unrestricted use, distribution, and reproduction in any medium, provided the original work is properly cited.

Background. Orthopedic procedures are among the commonest surgeries in the elderly. On the other hand, those patients have many risk factors suitable for development of postoperative complication due to prolonged postoperative recumbent that decrease by using anesthesia technique allowed early ambulation as far as possible. Aim of the Work. The aim of this study was to evaluate whether washout of the local anesthesia with $0.9 \%$ normal saline with bicarbonate through the epidural catheter could provide early ambulation throughout the faster recovery of the motor block. Patient and Methods. Sixty adult patients were included in this study and divided into three groups. In group I, epidural injection of bupivacaine was carried out without wash (control group). In group II, epidural injection of bupivacaine was followed by wash. In group III, epidural injection of both bupivacaine and nalbuphine was followed by wash. Results. They were significantly faster in both group II and group III as compared with group I. In group I, sensory recovery was insignificantly faster than the motor recovery. In group II motor recovery was significantly faster than sensory recovery. In group III motor recovery was significantly faster than the sensory recovery. Conclusions. These results suggested that early ambulation with an adequate postoperative analgesia was reported in patient receiving epidural injection of both bupivacaine and nalbuphine.

\section{Introduction}

Epidural analgesia and anesthesia were proved to be an excellent method of anesthesia in patients undergoing lower limb orthopedic operations, especially in patient with high risk to develop postoperative complication either from delay recovery of motor function or from use of large dose of narcotics IM or IV to achieve adequate sensory block [1].

Wash of epidural catheter with normal saline with or without $\mathrm{NaHCO}_{3}$ aims to achieve early motor recovery in a trial to avoid rapid sensory recovery as rapid motor recovery after wash of epidural catheter, nalbuphine was added as narcotic analgesic, and especially nalbuphine hydrochloride exhibits a ceiling effect in which the increase in the dose greater than $30 \mathrm{mg}$ does not produce further respiratory depression in the absence of other CNS active medications affecting respiration. In addition, prolonged postoperative sensory and motor block following neuraxial blocks (spinal or epidural block) after short surgical procedures is a major disadvantage of this technique [2]. Our study planned to use nalbuphine with local anesthesia.

As we worried about wash of epidural catheter with saline solution that results in faster return of the pain to the patient postoperatively, we aimed to study if adding nalbuphine to the local anesthetic agents can preserve the analgesic effect even after wash of local anesthesia. On the other hand, using narcotic with epidural anesthesia (IV or IM injected) requires large dose that may be associated with systemic complication; this disadvantage is minimized by injection of narcotics in epidural catheter. On the other hand, epidural injection of narcotic may be associated with complication, but this side effect is uncommon and occur with nalbuphine due to its ceiling effect, also using NSAIDs may not be suitable in elderly patient to avoid renal complication. Delay in the motor function in the major orthopedic surgery carries the risk of DVT development so epidural analgesia allows early 
mobilization, reduces the risk of DVT, and allows better cooperation with chest physiotherapy with preventing chest infections [3]. In addition, prolonged immobility has been associated with joint mobility restrictions, muscle weakness, critical illness neuromyopathies, pressure ulcers, DVT, and psychological disturbances [4]. Early ambulation has many advantages for patients as it promotes the maximal level of functional independence before discharge, decreased risk of neuromuscular abnormalities as muscle atrophy, nerve damage, slide ulcer, contracture, compartmental syndrome, and physical impairments and hospital acquired diseases as infections like acquired pneumonia. A normal saline bolus administered before discontinuation of the epidural catheter was reported to hasten the return of motor function following epidural anesthesia [5]. Resolution of epidural anesthesia depends on the redistribution of local anesthetics from neural tissues and its elimination by systemic uptake. Redistribution can be enhanced by decreasing the concentration of residual local anesthetics in the epidural space [6]. Increasing the speed of recovery from epidural anesthesia will improve patient acceptance of epidural anesthesia as well as potential cost savings and decease risk of prolonged recumbence [7]. Our aim was to decrease the risk of prolonged recumbence and how much as possible as to preserve the sensory block in patient undergoing orthopedic lower limb surgery. The aim of the study is to demonstrate the significant effect of injection of normal saline with sodium bicarbonate on motor and sensory in epidural catheter prior to its removal in orthopedic lower limb surgery.

\section{Patients and Methods}

After approval of the local Ethics Committee of Minia University Hospital, informed written consent was obtained from patient. Our study includes 60 patients undergoing orthopedic lower limb surgery in Minia University Hospital.

2.1. Preoperative Assessment. Full medical history examination and physical examination (chest, heart, abdomen, and other systems) were carried out. Preoperative investigation was done in the form of complete blood count, bleeding time, clotting time, and platelet count to exclude the risk of bleeding tendency and any other specific investigations required were done. The patient was excluded from the study if the platelets count was less than 100.000 or due to prolonged bleeding and clotting time.

2.2. Patients. The study is a randomized not a blind study. 60 patients of both sexes were divided into three groups (each group contains 20 patients): group I: control group include patients received epidural isobaric bupivacin $0.5 \%$ without washout, group II: include patients received epidural isobaric bupivacin $0.5 \%$ and washout by sodium bicarbonate $0.4 \mathrm{~mL}$ in $8.4 \%$ with normal saline $14.6 \mathrm{~mL}$ after the end of the surgery [8], and group III: epidural bupivacaine (isobaric $0.5 \%$ ) and nalbuphine $20 \mathrm{mg}$ washout by sodium bicarbonate $0.4 \mathrm{~mL}$ in $8.4 \%$ with normal saline $14.6 \mathrm{~mL}$ after the end of the surgery [8].
2.3. Anesthetic Technique. Electrocardiogram, heart rate, and peripheral oxygen saturation were monitored continuously, and noninvasive blood pressure was measured at $5 \mathrm{~min}$ intervals preoperatively.

Lumbar epidural catheter placement was performed with the patient in the sitting position using an 18-gauge Tuohy needle inserted in the midline at the L3-4 intervertebral space with the loss of resistance to air technique.

Epidural catheters were advanced deeply $3-5 \mathrm{~cm}$ into the epidural space. Following a negative aspiration for blood or cerebrospinal fluid, patients are divided into 3 groups. Groups $\mathrm{A}$ and $\mathrm{B}$ receive $0.5 \%$ bupivacaine and group $\mathrm{C}$ receives bupivacaine and $20 \mathrm{mg}$ nalbuphine. Sufficient volume from 21 to $28 \mathrm{~mL}$ bupivacaine (isobaric $0.5 \%$ ) was administered to obtain analgesic level up to T8 level in all patients.

If the anesthetist estimated that the resulting sensory blocked at $15 \mathrm{~min}$ would not to be high enough (T8), additional dose was given. The level of anesthetic block was assessed using a blunt rounded pin end for pinprick sensation. Testing was performed by moving in the cranial direction and the level of highest dermatomes where the sensation that was perceived as not painful or cold was recorded. Surgery was started when complete motor and sensory were blocked. All patients will receive oxygen $5 \mathrm{~L} / \mathrm{min}$ via facemask and were positioned supine in the operating room. Motor blocked was assessed using modified bromage scale [9]. Intraoperative hemodynamics (heart rate, mean arterial blood pressure, and oxygen saturation by pulse oximeter) were assessed and recorded. During anesthesia and surgery, mean arterial blood pressure less than $20 \%$ was treated with fluid infusion and repeated dose of $5 \mathrm{mg}$ of ephedrine. Fluid therapy was standardized during and after surgery.

All epidural boluses were preceded by a negative aspiration for blood and CSF and were infused over a $1 \mathrm{~min}$ period. Assessments of motor and sensory block were performed in postoperative care unit until complete motor and sensory recovery.

\subsection{Parameters Assessed: The Following Parameters}

\section{Were Assessed}

2.4.1. Pain Scores. The intensity of postoperative pain after surgery was assessed until first analgesic requirement was recorded. Postoperative pain greater than 3 on visual analogue pain (VAS) treated with intravenous ketorolac $(30 \mathrm{mg})$ and postoperative pain scores were compared between patients in 3 groups [10]. In visual analogue pain score (VAS), score (0) denotes no pain and score (10) denotes worst pain imaginable.

2.4.2. Time of Complete Motor Block Recovery. It was assessed by bromage scale.

2.4.3. Hemodynamic Effects. Mean arterial blood pressure, heart rate, pre, intra and postoperatively oxygen saturation using pulse oximeter are recorded. 
TABLE 1: Demographic data.

\begin{tabular}{|c|c|c|c|c|c|c|}
\hline & \multirow{2}{*}{ Group A $(n=20)$} & \multirow{2}{*}{ Group B $(n=20)$} & \multirow{2}{*}{ Group C $(n=20)$} & \multicolumn{3}{|c|}{$P$ value } \\
\hline & & & & $A$ versus $B$ & A versus $C$ & B versus C \\
\hline \multicolumn{7}{|l|}{ Age } \\
\hline $\mathrm{M} \pm \mathrm{SD}$ & $55 \pm 9$ & $55 \pm 6.5$ & $58 \pm 7$ & 0.829 & 0.256 & 0.357 \\
\hline \multicolumn{7}{|l|}{ Sex } \\
\hline Male & $13(65 \%)$ & $14(70 \%)$ & $12(60 \%)$ & \multirow{2}{*}{0.736} & \multirow{2}{*}{0.744} & \multirow{2}{*}{0.507} \\
\hline Female & $7(35 \%)$ & $6(30 \%)$ & $8(40 \%)$ & & & \\
\hline \multicolumn{7}{|c|}{ Height $(\mathrm{cm})$} \\
\hline $\mathrm{M} \pm \mathrm{SD}$ & $167 \pm 4$ & $166 \pm 2$ & $166 \pm 3$ & 0.681 & 0.784 & 0.891 \\
\hline \multicolumn{7}{|l|}{ Weight (kg) } \\
\hline $\mathrm{M} \pm \mathrm{SD}$ & $79 \pm 10$ & $79 \pm 8$ & $77 \pm 8$ & 0.943 & 0.595 & 0.547 \\
\hline \multicolumn{7}{|l|}{ ASA } \\
\hline Class I & $10(50 \%)$ & $13(65 \%)$ & $15(75 \%)$ & \multirow{2}{*}{0.337} & \multirow{2}{*}{0.102} & \multirow{2}{*}{0.490} \\
\hline Class II & $10(50 \%)$ & $7(35 \%)$ & $5(25 \%)$ & & & \\
\hline
\end{tabular}

Data are expressed as means $\pm \mathrm{SD}$. No significant differences between the three groups ( $n$ : number).

As regards (1) age, (i) there was no significant change between group A and group $\mathrm{B}(P=0.829)$; (ii) there was no significant change between group A and group C $(P=0.256)$; (iii) there was no significant change between group $\mathrm{B}$ and group $\mathrm{C}(P=0.357)$. As regards $(2)$ sex, (i) there was no significant change between group A and group B $(P=0.736)$; (ii) there was no significant change between group A and group $\mathrm{C}(P=0.774)$; (iii) there was no significant change between group $\mathrm{B}$ and group $\mathrm{C}(P=0.507)$. As regards $(3)$ height, (i) there was no significant change between group A and group $\mathrm{B}(P=0.681)$; (ii) there was no significant change between group A and group B $(P=0.784)$; (iii) there was no significant change between group $\mathrm{B}$ and group $\mathrm{C}(P=0.891)$. As regards (4) weight, (i) there was no significant change between group A and group $\mathrm{B}(P=0.943)$; (ii) there was no significant change between group A and group $\mathrm{C}$ $(P=0.595)$; (iii) there was no significant change between group $\mathrm{B}$ and group $\mathrm{C}(P=0.547)$.

2.4.4. Other Parameters. Incidence of respiratory depression (less than $10 \mathrm{breath} / \mathrm{min}$ ), nausea, vomiting urine retention, and purities were noted and recorded.

2.5. Statistical Analysis of the Data. Input data were processed using computer-based software (SPSS) version 11 interface [11].

Recorded values were presented as means \pm standard deviation. Statistical analysis included parametric and nonparametric methods by one-way or two-way analysis of variance (ANOVA) among the 3 different groups. Categorical data were compared using Fisher's exact test. A value of $P<$ 0.05 was considered statistically significant.

\section{Result}

3.1. Demographic Data (Patient Characteristics). There were no statistically significant differences $(P<0.05)$ between three groups as regards age, weight, sex ratio, height, and ASA classification (Table 1).

\subsection{Hemodynamic and Oxygen Saturation}

3.2.1. Oxygen Saturation (\%). There were no significant changes in mean oxygen saturation (\%), heart rate (beat/min), and mean arterial blood pressure $(\mathrm{mmHg})$ at any time during the study periods inside each group or between the three groups.

\subsection{Onset of Action}

(i) There were no significant changes between group A $(21 \pm 1.86)$ and group B $(19.6 \pm 2.52)$.

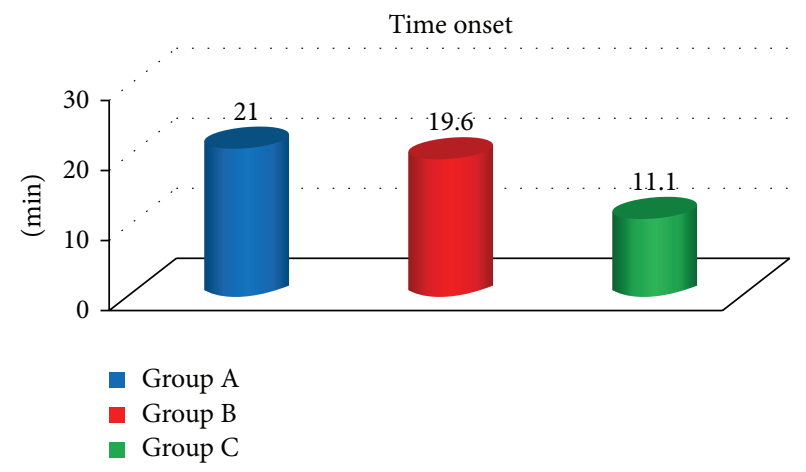

FIGURE 1: Onset of action in different groups expressed in minutes.

(ii) There were significant changes between group A (21 \pm $1.86)$ and group $\mathrm{C}(11.1 \pm 1.71)$.

(iii) There were no significant changes between group $\mathrm{B}$ $(19.6 \pm 2.52)$ and group C $(11.1 \pm 1.71)$ (Figure 1$)$.

3.4. Level of Sensory Block. There were no significant changes between three groups in the level of sensory block both at T6 and T8 (see Figure 2 and Table 2).

3.5. Score of Motor Block (Bromage Score). There were no significant changes between three groups in the bromage score of motor block (Figure 3).

\subsection{Offset of Anesthesia}

3.6.1. Motor Offset. After complete of surgery, patients in group $\mathrm{A}$ receive no bolus but patients in groups $\mathrm{B}$ and $\mathrm{C}$ 
TABLE 2: Level of sensory block.

\begin{tabular}{lccccc}
\hline Sensory level & Group A $(n=20)$ & Group B $(n=20)$ & Group C $(n=20)$ & A versus B & $\begin{array}{c}P \text { value } \\
\text { A versus C }\end{array}$ \\
\hline T8. & $18(90 \%)$ & $18(90 \%)$ & $17(85 \%)$ & 1.000 & 0.633 \\
T6. & $2(10 \%)$ & $2(10 \%)$ & $3(15 \%)$ & & 0.633 \\
\hline
\end{tabular}

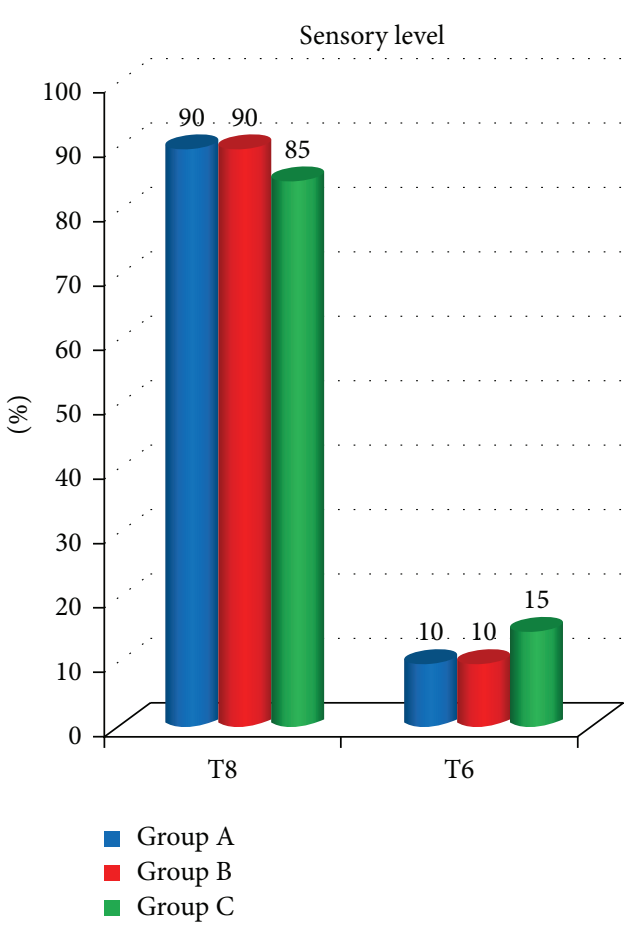

FIGURE 2: Score of sensory level.

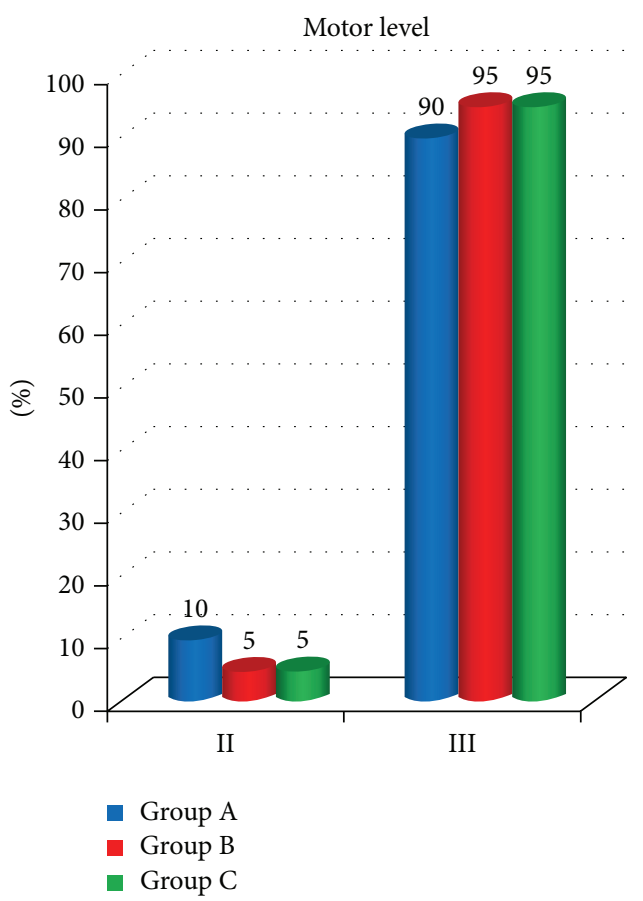

FIgURE 3: Score of motor level block.

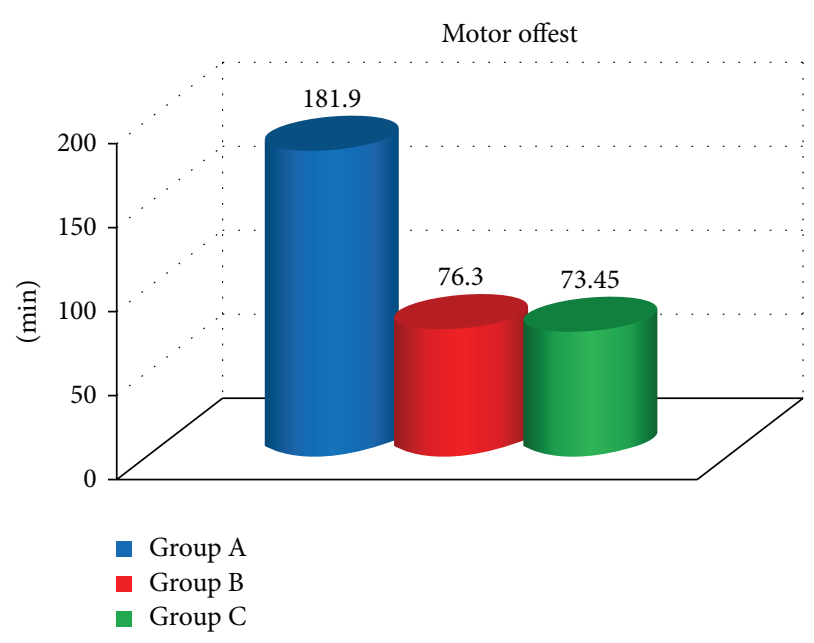

Figure 4: Motor offset expressed in minutes.

receive $14.6 \mathrm{~mL}$ normal saline with $0.4 \mathrm{~mL}$ sodium bicarbonate $8.4 \%$. According to the result, there were significantly changes between the groups. In group A, the patients have full motor strength ( $0=$ flexion of knee and feet $)$ after $181.9 \mathrm{~min} \pm$ 12.17 min. In group $B$, the patients have full motor strength $(0=$ flexion of knee and feet $)$ after $76.3 \mathrm{~min} \pm 6.2 \mathrm{~min}$. In group $\mathrm{C}$, the patients have full motor strength $(0=$ flexion of knee and feet) after $73.45 \mathrm{~min} \pm 9.94 \mathrm{~min}$. As regards the time of motor recovery, there are significant changes between group $\mathrm{A}$ and groups $\mathrm{B}$ and $\mathrm{C}$. There were significant changes between groups $\mathrm{B}$ and $\mathrm{C}$ (Figure 4).

3.6.2. Sensory Offset. There were insignificant changes in offset of sensory block between three groups (Figure 5).

3.7. Complications in the Studied Groups. Drowsiness and itching occurred in $10 \%$ of patients of group $\mathrm{C}$ and back pain in $25 \%$ of patients in group B with insignificance differences between studied groups; no other complications occurred during the study (Figure 6).

\section{Discussion}

Ambulatory anesthesia and surgical care have proven to be safe, convenient, and cost-effective and can be performed in a variety of facilities. The aim of an anesthesiologist in ambulatory setting is to use the most favorable anesthetic technique that would give excellent operating conditions, a rapid recovery, no postoperative side effects, and a high degree of patient satisfaction. Ambulatory anesthesia is tailored to meet 


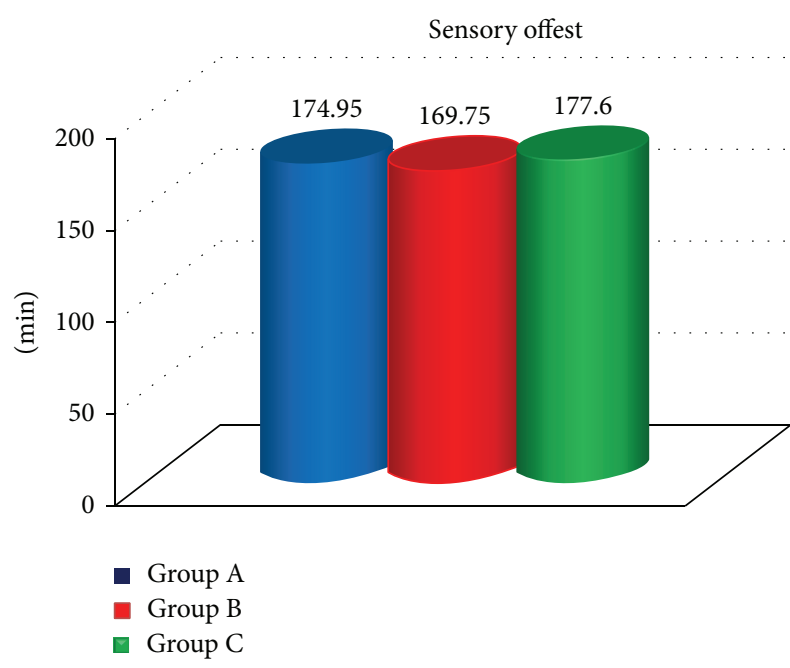

FIGURE 5: Sensory offset expressed in minutes.

the needs of ambulatory surgery so patients may go home soon after the operation. Postoperative side effects, which include pain, dizziness, headache, postoperative nausea and vomiting, and pruritus, when minimized or prevented will improve quality and time of recovery. Epidural nalbuphine proved to be an excellent method of anesthesia in patients undergoing orthopedic lower limb surgery.

Persistence of motor blockade after epidural anesthesia can be a source of dissatisfaction to both patients and recovery room staff especially in patients with high risk of DVT development [12].

Our study demonstrates that the addition of epidural nalbuphine will produce rapid onset of anesthesia and addition of normal saline with sodium bicarbonate can provide early ambulation. A synergistic effect has been shown to exist between local anesthetics and opioids administered intrathecally. This effect was demonstrated whether the opioid was given at the same time [13] or before the local anesthetic [14]. These effects were reported experimentally.

Clinical studies also demonstrate that this synergism also provides the advantages of significantly better pain relief, better analgesia, with lower concentration of the local anesthetic, shorter onset of analgesia, and increased duration of analgesia [15].

Our result showed that there is a significant change in the time of recovery of motor block in both groups $B$ and $C$ than in group A. In addition, there is a faster offset in groups A, B, and group $\mathrm{C}$. The mechanism by which epidural injections of crystalloid solutions can alter the neural blockade is unclear. Intensity of epidural blockade may lessen because crystalloid solutions dilute local anesthetics in or adjacent to the epidural space, decreasing the concentration of unbound drug at neuronal sites of action [16].

Alternatively, decreases in intensity could occur if crystalloid solutions enhanced clearance of local anesthetic from neural and connective tissues or from the subarachnoid and epidural spaces. Saline injection into the epidural space appears to augment both secretion and clearance of CSF and may therefore enhance elimination of local anesthetic from the subarachnoid space. Large volumes of fluid injected into the epidural space may also promote caudate and cephalic spread of drug, increasing surface area for uptake into blood vessels and lymphatics. Clearance of local anesthetics occurs via diffusion and uptake into lymph and blood vessels and hence greater vascular uptake and is influenced by physicochemical properties (molecular weight, pKa, lipid solubility, and protein binding) of local anesthetic agents and their effects on spinal and epidural blood flow. The Injection may dilute local anesthesia and decrease the concentration of drug available for binding at neuronal action sites [17].

But sensory block is preserved in three groups in contrast to the motor block in groups $\mathrm{A}, \mathrm{B}$, and $\mathrm{C}$. The above data can explained as by injection of saline in the epidural space, the result is dilution of bupivacine and more persistent sensory block than the motor block; these result in preferential sensory block as observed in our study because the drug at lower concentration will produce sensory block and at higher concentration can produce motor block [18].

We add sodium bicarbonate with normal saline because normal saline $0.9 \%$ is slightly acidic at a $\mathrm{PH}$ of 5.0 and we could lower the $\mathrm{PH}$ of bupivacaine which would result in less redistribution from epidural space. So we use sodium bicarbonate to increase the concentration of nonionized free base and more rapid diffusion rate of the drug to adjacent tissues, including blood and resulting in high peak levels [19]. Our study agrees with the study of Johnson et al. [16] that reported injection of $45 \mathrm{~mL}$ epidural bolus to each of the treatment groups (normal saline or lactated ringer's solution) but did so in $15 \mathrm{~mL}$ aliquots separated by 15 minutes. They found that the $45 \mathrm{~mL}$ bolus hastened the resolution of motor but not sensory blockade after $0.75 \%$ bupivacaine for cesarean delivery.

This amount of saline cannot be used in the injection in elderly individuals as in these individuals, the areolar tissues become dense and firm, partially sealing the intervertebral foramina and the epidural space also becomes more compliant and less resistant because of the degenerated fatty tissue and the reduced contents appear with the advancing age. In addition, progressive changes of the spinal column associated with aging have been postulated to produce a narrowing of the intervertebral foramina along the column [20].

Another study of Sitzman et al. [19] showed the effect of two postoperative boluses of $15 \mathrm{~mL}$ of epidural saline separated by 30 minutes after administration of $2 \%$ lidocaine with 1:200,000 epinephrine for obstetrical and gynecologic surgery. They found that the two boluses injection as compared with no epidural saline bolus injection as a control significantly reduced the time of full motor recovery and sensory recovery.

Similarly, Park et al. [6] reported that a $30 \mathrm{~mL}$ bolus of saline hastened motor and sensory recovery in elderly men who received $12 \mathrm{~mL}$ of $2 \%$ lidocaine with $50 \mu \mathrm{g}$ of fentanyl epidurally for transurethral surgery.

The study of Chan et al. [17] showed a dose-response study with evaluation of six volunteers sensory and motor recovery with three different saline volumes. Each subject received plain $2 \%$ lidocaine followed 30 minutes later by a $1 \mathrm{~mL}, 20 \mathrm{~mL}$, or $40 \mathrm{~mL}$ normal saline bolus. They found that 


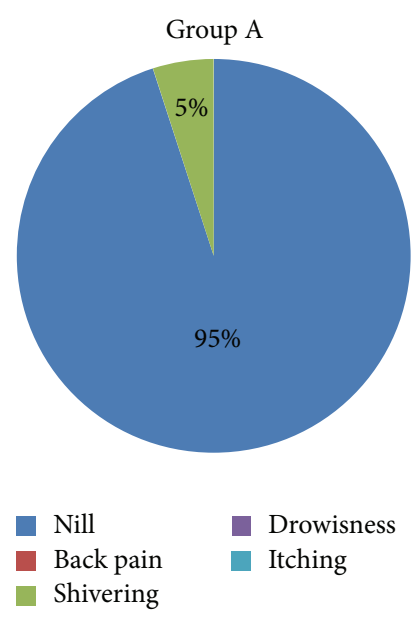

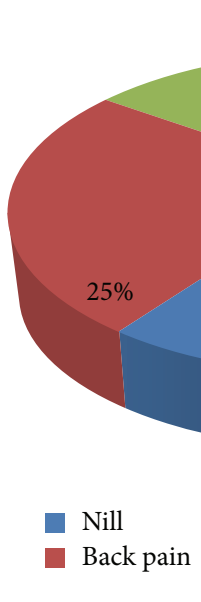

Group B

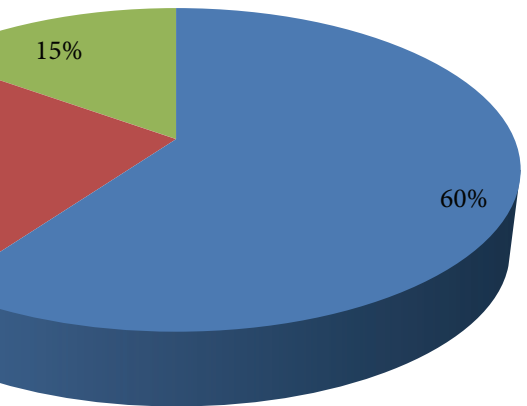

- Shivering

Drowisness

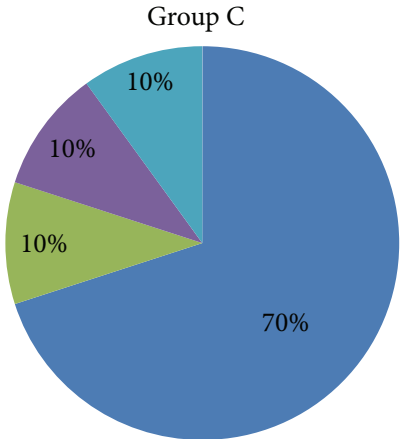

Nill
Back pain
Shivering

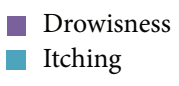

FiguRe 6

subjects who received the $40 \mathrm{~mL}$ normal saline bolus had significantly faster recovery of sensory and motor function compared with the $20 \mathrm{~mL}$ and $1 \mathrm{~mL}$ receiving groups. These results suggest that high volume may hasten recovery of motor and sensory function.

According to the VAS the first analgesic requirement is the same in groups A, B, and C. Our study demonstrates no wash occurring in nalbuphine because nalbuphine-like fentanyl is lipophilic that it sticks to fatty compartments found in the epidural space and not affected by hydrophilic washout saline. These results were similar to that of Park et al. [6] who reported that epidural saline washout does not affect the epidural fentanyl injection.

In the present study, there were no significant differences between all groups in respect to changes in MAP, heart rate, and oxygen saturation. In this study shivering occurs in 5\% of patient in group A, $15 \%$ in group B, and $10 \%$ in group C. Intra-anesthetic and postanesthetic shivering etiology may be related to the inhibition of medullary reflexes, regional sympathetic activity block that leads to peripheral vasodilatation, and heat loss and pyrogenic substances release among other causes [21].

This may be explained by addition of large volumes of saline that result in overstretch of epidural membrane with back pain, especially in elder patients included in this study with collapsed epidural space. On the other hand, this pain was not occurring in group $\mathrm{C}$ that also received the same volume of saline; this may be due to coadministration of nalbuphine.

In elderly individuals, the areolar tissues become dense and firm, partially sealing the intervertebral foramina. The epidural space also becomes more compliant and less resistant because of degenerated fatty tissue and reduced contents with advancing age. In addition, progressive changes of the spinal column associated with aging have been postulated to produce narrowing of the intervertebral foramina along the column [20].

1st analgesic requrements when VAS was equal to 3; there were no complications from ketorolac. Drowsiness occur in
$10 \%$ of patients in group $\mathrm{C}$ as a complications. The sedation usually results from cephalic migration of opioids in the CSF and also blood level attained with the lipid soluble drugs as sufentanil [22].

We planned for early recovery of motor activities to prepare the patient for early ambulation that may take place in the form of exercising calf muscles and stretching the legs while the patient is in the sitting position, raising and lowering the heels while keeping the toes on the floor or raising and lowering the toes while keeping the heels on the floor as presented in Voronkov et al. [23] study. We were not considering early ambulations by early discharge from postoperative care unite but early ambulation that enable patient to do calf muscle exercise.

Intra-anesthetic and postanesthetic shivering etiology may be related to the inhibition of medullary reflexes, regional sympathetic activity block leading to peripheral vasodilatation, and heat loss and pyrogenic substances release among other causes. In study of Walmsley et al. [24], 30 patients undergoing postpartum tubal ligation under extradural anesthesia initially received bupivacaine at $4^{\circ} \mathrm{C}$, and the incidence of shivering was $47 \%$. Further bupivacaine warmed to $41^{\circ} \mathrm{C}$ was injected into 8 patients in whom the resultant shivering was marked. In four of these patients, the shivering stopped. The authors concluded that thermosensitive tissue within the spinal canal contributes to the shivering observed in association with extradural anesthesia. This finding confirms our findings in this study.

\section{Conclusion}

Postoperative injection of normal saline with sodium bicarbonate via epidural catheter markedly shortened the duration of motor of motor blockade resulting from epidural anesthesia with bupivacaine $0.5 \%$ used for orthopedic lower limb surgery. Addition of nalbuphine to epidural bupivacaine can provide adequate block with good analgesia. 


\section{Conflict of Interests}

The authors declare that there is no conflict of interests regarding the publication of this paper.

\section{Authors' Contribution}

All authors contributed equally to this paper.

\section{Acknowledgment}

The abstract of this paper was only represented as e-poster in 33rd Annual European Society of Regional Anaesthesia \& Pain Therapy (ESRA) that was held in last September.

\section{References}

[1] R. Barker, A. Schiferer, C. Gore et al., "Femoral nerve blockade administered preclinically for pain relief in severe knee trauma is more feasible and effective than intravenous metamizole: a randomized controlled trial," The Journal of Trauma, vol. 64, no. 6, pp. 1535-1538, 2008.

[2] A. Mukherjee, A. Pal, J. Agrawal, A. Mehrotra, and N. Dawar, "Intrathecal nalbuphine as an adjuvant to subarachnoid block: what is the most effective dose?" Anesthesia: Essays and Researches, vol. 5, no. 2, pp. 171-175, 2011.

[3] M. E. Hecht, A Practical Guide to Hip Surgery: From Pre-Op to Recovery, 2010.

[4] P. E. Morris, L. Griffin, M. Berry et al., "Receiving early mobility during an intensive care unit admission is a predictor of improved outcomes in acute respiratory failure," The American Journal of the Medical Sciences, vol. 341, no. 5, pp. 373-377, 2011.

[5] D. Guryay, G. T. Karaege, K. Katircioglu, M. Y. Ozkalkanli, U. Ozgurbuz, and S. Savaci, "The effects of an epidural infusion of ropivacaine versus saline on sensory block after spinal anesthesia," Regional Anesthesia and Pain Medicine, vol. 33, no. 3, pp. 217-221, 2008.

[6] E. Y. Park, H. K. Kil, W. S. Park, N.-H. Lee, and J.-Y. Hong, "Effect of epidural saline washout on regression of sensory and motor block after epidural anaesthesia with $2 \%$ lidocaine and fentanyl in elderly patients," Anaesthesia, vol. 64, no. 3, pp. 273276, 2009.

[7] D. A. Raw, J. K. Beattie, and J. M. Hunter, "Anaesthesia for spinal surgery in adults," British Journal of Anaesthesia, vol. 91, no. 6, pp. 886-904, 2003.

[8] T. J. Coderre, J. Katz, A. L. Vaccarino, and R. Melzack, "Contribution of central neuroplasticity to pathological pain: review of clinical and experimental evidence," Pain, vol. 52, no. 3, pp. 259-285, 1993.

[9] A. C. Graham and J. H. McClure, "Quantitative assessment of motor block in labouring women receiving epidural analgesia," Anaesthesia, vol. 56, no. 5, pp. 470-476, 2001.

[10] G. A. Hawker, S. Mian, T. Kendzerska, and M. French, "Measures of adult pain: Visual Analog Scale for Pain (VAS Pain), Numeric Rating Scale for Pain (NRS Pain), McGill Pain Questionnaire (MPQ), Short-Form McGill Pain Questionnaire (SF-MPQ), Chronic Pain Grade Scale (CPGS), Short Form-36 Bodily Pain Scale (SF-36 BPS), and measure of Intermittent and Constant Osteoarthritis Pain (ICOAP)," Arthritis Care \& Research, vol. 63, supplement 11, pp. S240-S252, 2011.
[11] R. Lévesque, SPSS Programming and Data Management: A Guide for SPSS and SAS Users, 4th edition, 2007.

[12] J. G. Brock-Utne, A. Macario, M. F. Dillingham, and G. S. Fanton, "Postoperative epidural injection of saline can shorten postanesthesia care unit time for knee arthroscopy patients," Regional Anesthesia and Pain Medicine, vol. 23, no. 3, pp. 247251, 1998.

[13] D. M. Pöpping, N. Elia, E. Marret, M. Wenk, and M. R. Tramr, "Opioids added to local anesthetics for single-shot intrathecal anesthesia in patients undergoing minor surgery: a metaanalysis of randomized trials," Pain, vol. 153, no. 4, pp. 784-793, 2012.

[14] B. Akerman, E. Arwestrom, and C. Post, "Local anesthetics potentiate spinal morphine antinociception," Anesthesia and Analgesia, vol. 67, no. 10, pp. 943-948, 1988.

[15] G. McLeod and C. Cumming, "Thoracic epidural anaesthesia and analgesia," Continuing Education in Anaesthesia, Critical Care \& Pain, vol. 4, no. 1, pp. 16-19, 2004.

[16] M. D. Johnson, G. A. Burger, P. S. Mushlin, G. R. Arthur, and S. Datta, "Reversal of bupivacaine epidural anesthesia by intermittent epidural injections of crystalloid solutions," Anesthesia and Analgesia, vol. 70, no. 4, pp. 395-399, 1990.

[17] V. W. Chan, S. Nazarnia, Z. Kaszas, and A. Perlas, “The impact of saline flush on regression of sensory and motor block after epidural administration with $2 \%$ lidocaine and fentanyl in elderly patients," Anesthesia, vol. 64, pp. 273-267, 2009.

[18] T. Nishiyama, "Comparison of the motor and sensory block by ropivacaine and bupivacaine in combination with lidocaine in interscalene block," Medicinski Arhiv, vol. 66, no. 5, pp. 315-317, 2012.

[19] B. T. Sitzman, C. A. DiFazio, P. A. Playfair et al., "Reversal of lidocaine with epinephrine epidural anesthesia using epidural saline washout," Regional Anesthesia and Pain Medicine, vol. 26, no. 3, pp. 246-251, 2001.

[20] Y. Hirabayashi, R. Shimizu, I. Matsuda, and S. Inoue, "Effect of extradural compliance and resistance on spread of extradural analgesia," British Journal of Anaesthesia, vol. 65, no. 4, pp. 508513,1990

[21] E. Silva and D. Quinto, "Controle da Hipotermia Acidental," in Atualização em Anestesiologia, A. M. Yamashita, Ed., pp. 111-129, Office Editora, São Paulo, Brazil, 1999.

[22] M. A. Chaney, "Side effects of intrathecal and epidural opioids," Canadian Journal of Anaesthesia, vol. 42, no. 10, pp. 891-903, 1995.

[23] M. Voronkov, D. Ocheret, S. Bondarenko, Y. I. Yu, and S. Koren, "Administration of nalbuphine to heroin addicts. Feasibility and short-term effects," Heroin Addiction and Related Clinical Problems, vol. 10, no. 1, pp. 19-24, 2008.

[24] A. J. Walmsley, A. H. Giesecke, and J. M. Lipton, "Contribution of extradural temperature to shivering during extradural anaesthesia," British Journal of Anaesthesia, vol. 58, no. 10, pp. 1130$1134,1986$. 


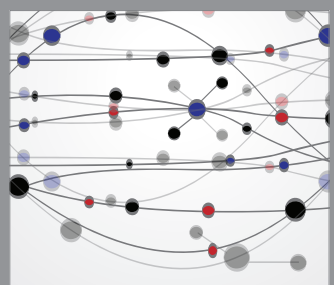

The Scientific World Journal
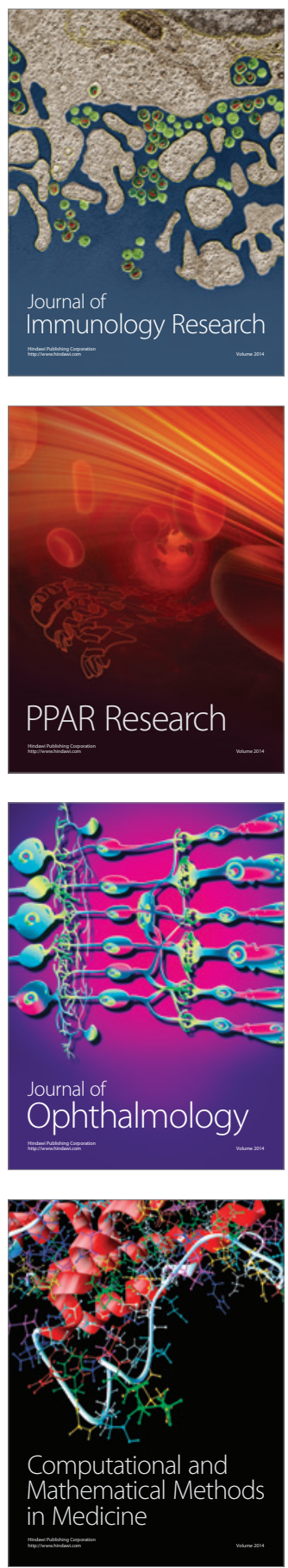

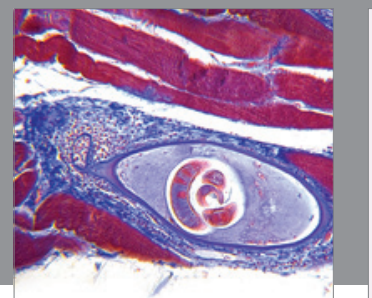

Gastroenterology

Research and Practice
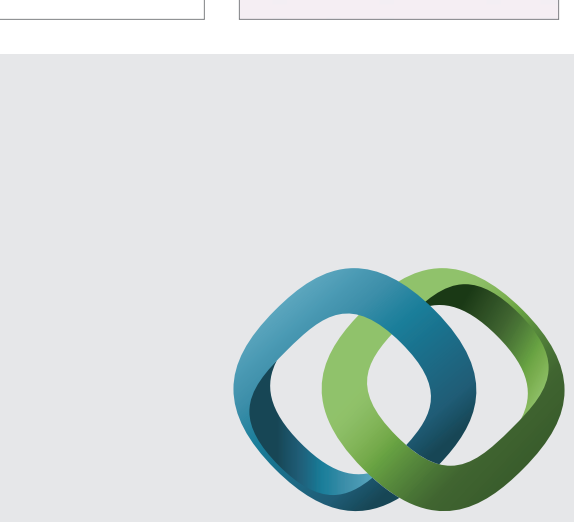

\section{Hindawi}

Submit your manuscripts at

http://www.hindawi.com
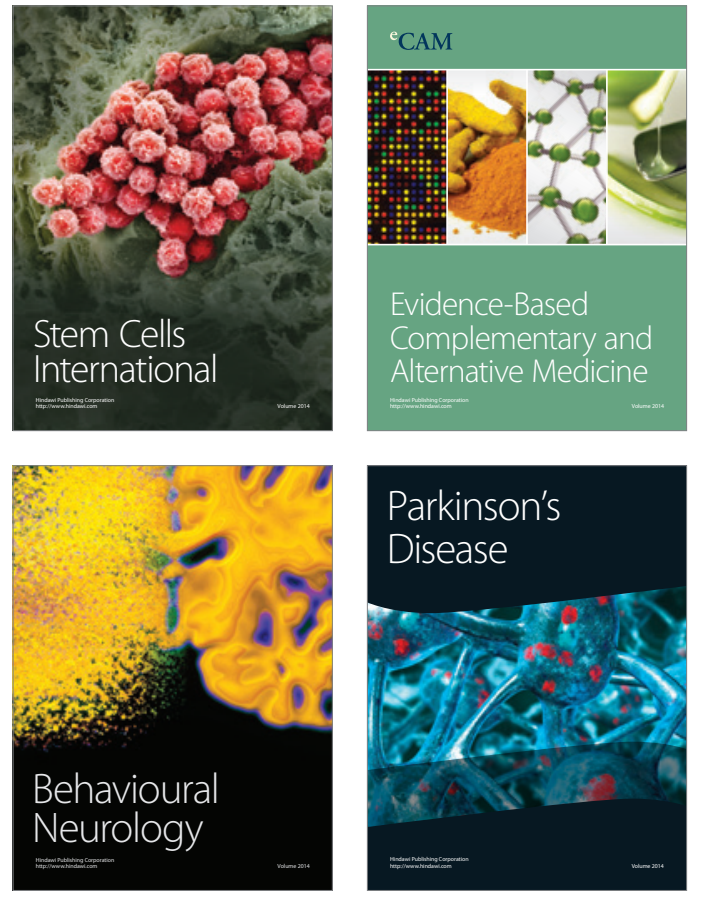
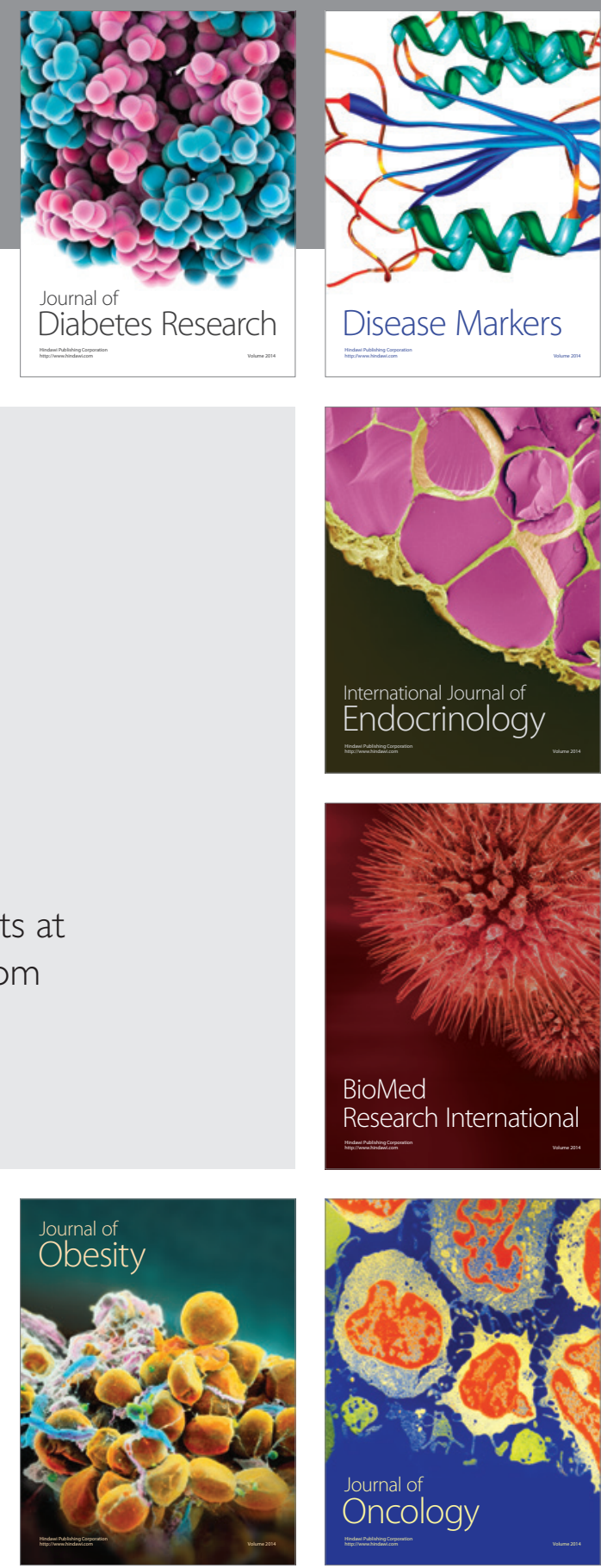

Disease Markers
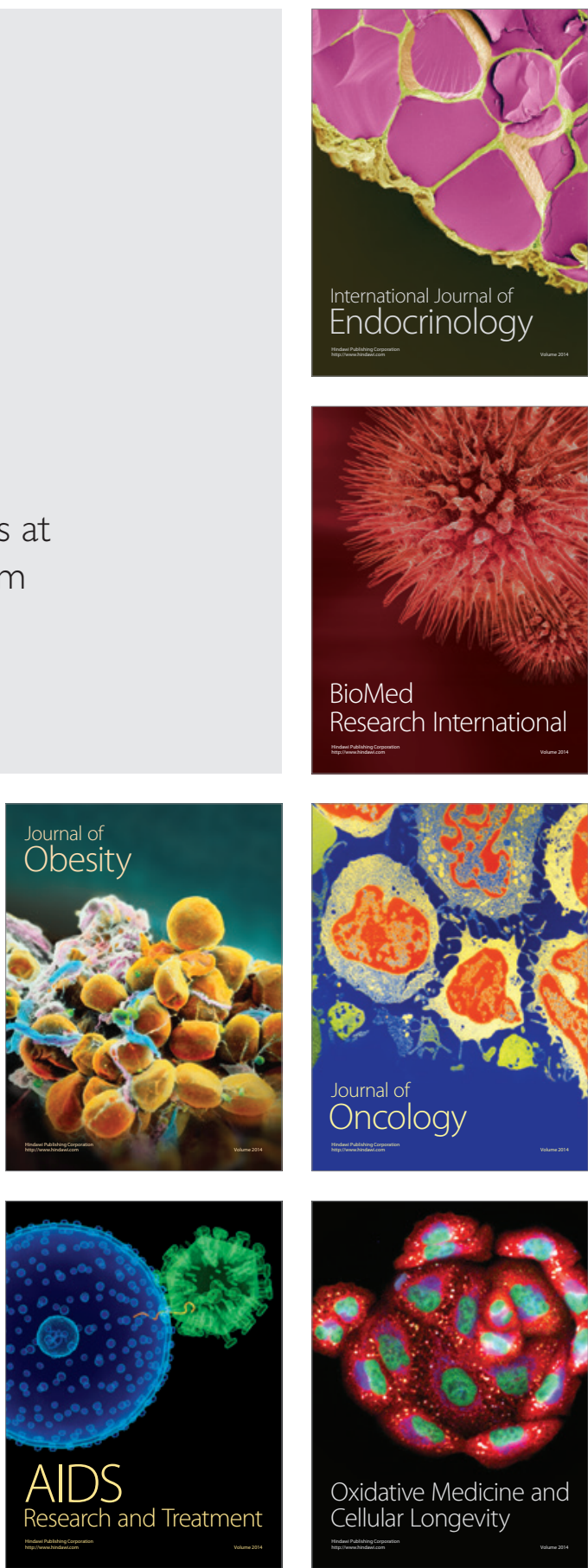